\title{
Suaeda maritima-based herbal coils and green nanoparticles as potential biopesticides against the dengue vector Aedes aegypti and the tobacco cutworm Spodoptera litura
}

\begin{abstract}
The overuse of synthetic pesticides to control insect pests leads to physiological resistance and adverse environmental effects, in addition to high operational cost. Insecticides of botanical origin have been reported as useful for control of agricultural and public health insect pests. This research proposed a novel method of mangrove-mediated synthesis of insecticidal silver nanoparticles (AgNP) using Suaeda maritima, acting as a reducing and stabilizing agent. AgNP were characterized by UV-vis spectroscopy, Fourier transform infrared (FTIR) spectroscopy, scanning electron microscopy (SEM), energy-dispersive X-ray spectroscopy (EDX), and Xray diffraction (XRD) analysis. S. maritima aqueous extract and mangrove-synthesized AgNP showed larvicidal and pupicidal toxicity against the dengue vector Aedes aegypti and the tobacco cutworm Spodoptera litura. In particular, LC50 of AgNP ranged from 8.668 (larva I) to 17.975 ppm (pupa) for A. aegypti, and from 20.937 (larva I) to $46.896 \mathrm{ppm}$ (pupa) for S. litura. In the field, the application of S. maritima extract and AgNP $(10 \times$ LC50) led to 100\% mosquito larval reduction after $72 \mathrm{~h}$. Smoke toxicity experiments conducted on A. aegypti adults showed that S. maritima leaf-, stem- and root-based coils evoked mortality rates comparable or higher if compared to permethrin-based positive control $(62 \%, 52 \%, 42 \%$, and 50.2 respectively). In ovicidal experiments, egg hatchability was reduced by $100 \%$ after treatment with $20 \mathrm{ppm}$ of AgNP and $250 \mathrm{ppm}$ of S. maritima extract. Furthermore, low doses of the AgNP inhibited the growth of Bacillus subtilis, Klebsiella pneumoniae and Salmonella typhi. Overall, our results highlighted the potential of S. maritima-based herbal coils and green nanoparticles as biopesticides in the fight against the dengue vector A. aegypti and the tobacco cutworm S. litura.
\end{abstract}

Keyword: Arbovirus; Crop protection; Mangrove; Antibacterial activity; Nanotechnology 